\title{
The importance of catastrophizing for successful pharmacological treatment of peripheral neuropathic pain
}

This article was published in the following Dove Press journal:

Journal of Pain Research

24 June 2014

Number of times this article has been viewed

\section{Cory Toth \\ Shauna Brady \\ Melinda Hatfield \\ Department of Clinical \\ Neurosciences, University of Calgary, \\ Calgary, AB, Canada}

Correspondence: Cory Toth

Room 155, University of Calgary,

Department of Clinical Neurosciences,

3330 Hospital Drive NW, Calgary,

$\mathrm{AB}$, Canada, T2N4NI

$\mathrm{Tel}+\mid 403220883$ I

Fax + I 403283873 |

Email corytoth@shaw.ca
Objective: Catastrophizing may be a negative predictor of pain-related outcomes. We evaluated the impact of catastrophizing upon success of first-line pharmacotherapy in the management of neuropathic pain $(\mathrm{NeP})$ due to peripheral polyneuropathy.

Methods: Patients with confirmed NeP with NeP Visual Analog Scale (VAS) pain severity score $\geq 4$ (0-10 scale) completed the Coping Strategies Questionnaire (CSQ) catastrophizing subscale at baseline. Pharmacological therapy consisting of first-line agents gabapentin, pregabalin, or a tricyclic antidepressant was initiated. Other measures examined included the Karnofsky Performance Scale, Beck Depression Inventory, EuroQol Quality of Life Health Questionnaire, and Modified Brief Pain Inventory. At 3 and 6 months, questionnaires were repeated and adverse effect reporting was completed. Outcome measures assessed were pharmacotherapy success ( $\geq 30 \%$ relief of $\mathrm{NeP}$ ) and tolerability over 6 months of follow-up. Bivariate relationships using Pearson product-moment correlations were examined for baseline CSQ catastrophizing subscale score and the change in the NeP VAS scores and medication discontinuation.

Results: Sixty-six patients were screened, 62 subjects participated, and 58 subjects (94\%) completed the final follow-up visit. Greater catastrophizing was associated with poor pain relief response and greater likelihood of discontinuation of pharmacotherapy, reports of greater disability, and impaired quality of life. Duration of pain was negatively associated with likelihood of pharmacotherapy success.

Conclusion: Catastrophizing exerts maladaptive effects on outcomes with pharmacotherapy in $\mathrm{NeP}$ patients. Detection of catastrophizing during clinical visits when pharmacological therapy is being considered can be a predictive factor for patient outcomes.

Keywords: neuropathic pain, catastrophizing, coping, pharmacotherapy

\section{Introduction}

Neuropathic pain $(\mathrm{NeP})$ is a form of chronic pain caused by a lesion or disease of the somatosensory nervous system. ${ }^{1} \mathrm{NeP}$ negatively impacts upon quality of life in several domains, also contributing to concomitant mood and sleep disorders. ${ }^{2-5}$ Unfortunately, $\mathrm{NeP}$ is often refractory to management with many NeP patients not achieving therapeutic efficacy and tolerability with use of standard pharmacological therapies. However, some patients with $\mathrm{NeP}$ have good outcomes with minimal therapies. The reasons for this discordance are not clear, but may relate to factors including genetics, pharmacokinetics, ability to achieve appropriate dosing, ${ }^{6,7}$ presence of comorbidities and economic burden, ${ }^{8}$ or even culture and religion. ${ }^{9,10}$

Psychological factors can also impact significantly upon chronic pain and its experience. ${ }^{11,12}$ This is not surprising, as pain is frequently accompanied by strong 
emotions as well as somatogenic and psychological factors. ${ }^{13}$ In many situations, coping techniques are required to deal with these issues. Defined as the ability to manage stressful situations believed to surpass an individual's capabilities or resources, ${ }^{14}$ coping can consist of different responses including: 1) attempts to control a stressor, such as with problem solving; 2) social resource management, such as with seeking social supports; and 3) adapting one's goals, such as with acceptance. ${ }^{15,16}$ While some individuals persist with goal attainment despite chronic pain, others will engage in bed rest and avoidance; these behaviors depend on the presence of different skill sets and specific beliefs about the nature of pain and its management. For patients with chronic pain, coping efforts may involve both positive and negative outcomes depending on chronic pain severity and its comorbidities. For example, some active coping strategies have been effective, ${ }^{17,18}$ including prayer and hoping strategies. ${ }^{19}$ The use of these positive coping techniques is associated with less severe pain, less depressive symptoms, less functional impairment, and higher self-efficacy. In contrast, passive coping techniques such as avoidance and escape correlate with worsening mood, increased pain severity or flares of pain, greater functional impairment, and lower general self-efficacy. ${ }^{20}$

Catastrophizing, another form of negative coping strategy frequently used by chronic pain patients, is one of the strongest psychological predictors for worsening of pain experience. ${ }^{21}$ Defined as "an exaggerated negative mental set brought to bear during actual or anticipated painful experience", pain catastrophizing may contain dysfunctional foci contributing to three separate but related dimensions of appraisal composing catastrophizing: rumination ("I worry all the time about whether it will end"), magnification ("It is terrible and I feel it is never going to get any better"), and helplessness ("I feel like I can't go on"). ${ }^{22,23}$ The extent of pain positively relates to levels of catastrophizing in several clinical populations. ${ }^{12,21,22}$ As well, catastrophizing can be associated with increased chronic pain-related disability after injury ${ }^{24}$ or in other chronic diseases, ${ }^{25}$ perhaps related to greater pain severities reported in high catastrophizers. ${ }^{26,27}$ Indeed, catastrophizing may have closer associations with pain-related disability than seen with other comorbidities, such as with depression. ${ }^{28,29}$ When concurrently present with mental health conditions, the presence of catastrophizing reduces the efficacy of pain-related interventions. ${ }^{30-32}$ The efficacy of physical therapy interventions for musculoskeletal injury, diagnosed as soft tissue injury or whiplash injury, declines with higher Pain Catastrophizing Scale scores. ${ }^{30,32}$
In fact, catastrophizing levels at baseline predict later pain levels after oral pharmacotherapy intervention. ${ }^{33}$ For patients with various $\mathrm{NeP}$ conditions, the therapeutic response of topical 2\% amitriptyline and 1\% ketamine weakened with higher scores on the Pain Catastrophizing Scale. ${ }^{31}$ Despite knowledge to date, there remains uncertainty regarding the relationship of catastrophizing with pharmacotherapy interventions and the impact of catastrophizing management outcomes in patients with $\mathrm{NeP}^{22}$ As a result, we do not know if baseline catastrophizing levels predict outcomes with the most commonly used intervention for NeP, first-line oral pharmacotherapies. We are not aware of studies examining pharmacotherapy compliance in high catastrophizing pain patients, but catastrophizing can certainly impact upon self-management adherence in chronic pain patients. ${ }^{34}$ As a result, we sought to determine the role of catastrophizing upon pharmacological outcomes of pain relief success, tolerability, and therapy continuation in a population of patients with peripheral neuropathy-associated NeP.

The aim of this study was to determine the impact of catastrophizing as a maladaptive coping factor that could predict the outcome of guideline-mandated pharmacotherapy ${ }^{35}$ efficacy and tolerability in patients with $\mathrm{NeP}$ due to peripheral polyneuropathy. A successful outcome was defined as the presence of sufficient pain relief ( $\geq 30 \%$ improvement in pain severity) without medication discontinuation due to intolerance or perceived inefficacy. We hypothesized that catastrophizing would be a predictor of poor medication response and tolerability due to inherent features of catastrophizing such as helplessness and magnification. Our secondary objectives were to determine the impact of catastrophizing upon ancillary measures of mood, anxiety, sleep, patient satisfaction, and patient global impression of change in their condition.

\section{Materials and methods Patient assessment}

We prospectively evaluated patients with $\mathrm{NeP}$ at a tertiary care NeP clinic in Calgary, AB, Canada. ${ }^{36}$ This investigation was designed as a prospective cohort assessment as part of the patients' regular clinical care. All patients enrolled in this study had provided informed written consent for the assessment of their clinical outcomes using questionnaires performed at regular follow-up visits, as approved by the local health research ethics board (Conjoint Health Research Ethics Board, University of Calgary). All patients completed questionnaires prior to their clinical assessment on the first day of participation. 
The DN4 questionnaire ${ }^{37}$ was used to confirm the presence of $\mathrm{NeP}$ - inclusion required a score of $\geq 4$. When another coincidental cause for chronic pain was present, enrollment was permitted if, in the opinion of the enrolling neurologist, this condition could easily be separated from the NeP condition and if it was not the cause of the most severe chronic pain.

Patient care within the NeP clinic included noninvasive assessment and management of NeP. Focus was placed upon improvement in patient function and quality of life in all cases, with pharmacological intervention, physical and exercise therapy, family and social counseling, patient education, and vocational counseling offered. There was no formal psychological counseling provided, although it is possible that some patients may have received this of their own accord. Participation in forms of therapy was neither inclusive nor mandatory, and was variable between patients. All patients had a complete neurological examination performed. Diagnosis of polyneuropathy etiology was performed as previously described. ${ }^{38}$ During each clinic visit, clinical, psychological, and overall well-being assessments were performed, and education and counseling were provided as needed regarding the patient's condition, its prognosis, and its management options. Management decisions were made in tandem by physician and patient at the conclusion of the first visit. Patients were seen at 0,3 , and 6 months for clinical assessment and management, completion of surveys and questionnaires, and other non-pharmacological forms of chronic pain care.

Inclusion criteria consisted of: $\geq 18$ years of age; diagnosis of peripheral polyneuropathy with associated $\mathrm{NeP}$; $\geq 6$ months duration of NeP; NeP severity was $\geq 4$ on the Visual Analog Scale (VAS), with severity enough to require the prescription of a first-line pharmacological agent; patient agreement to attend scheduled clinical appointments in 3 and 6 months' time; and completion of informed consent. Exclusion criteria consisted of: diagnosis of another cause of NeP other than peripheral polyneuropathy; current use of a first-line pharmacotherapy or prior use of all possible first-line pharmacotherapies; presence of other serious health concerns that may have interfered with the follow-up visits; inability to provide information assessed in questionnaires due to language or cognitive issues; and presence of another cause of chronic pain of greater severity than that of NeP due to peripheral polyneuropathy which could interfere with assessments. Patients with concurrent tolerable musculoskeletal pain, such as with osteoarthritis, were not excluded if in the opinion of the investigator this condition would not be expected to interfere with data collection and follow-up visits.

\section{Pharmacotherapy intervention}

Following completion of questionnaires at the baseline visit, prescription of a first-line NeP pharmacotherapy (amitriptline, nortriptyline, gabapentin, pregabalin) occurred using the Canadian Pain Society Consensus Statement and Guidelines. ${ }^{39}$ The medication was selected by the physician and patient through consensus after accounting for the patients' demographics and comorbidities. There were no attempts to use one of these first-line agents more than any other agent. If a patient had already received or was intolerant to each of the four medications (and the two classes of medications [tricyclic antidepressants or gabapentinoids]), then that patient was excluded from further study. For patients where at least one of these medications had been previously attempted, only those first-line medications for which the patient was naïve were considered. Dosing of the selected agent took place on an individual basis based upon pain severity, comorbidities, body weight, age, and other potential factors. Regular use of pharmacotherapy for the 6 months preceding the last follow-up visit was recorded.

\section{Primary outcome measure}

We assessed for tolerability and efficacy of pharmacotherapy as the primary outcome measure. Definition of a successful pharmacotherapeutic intervention was dependent upon adequate pain relief in the absence of medication discontinuation. For the determination of successful pain relief, we used a threshold of $\geq 30 \%$ pain relief at 3 or 6 months based on the baseline pain VAS score for pain severity recorded pre-intervention. The VAS was determined by measuring an unmarked $100 \mathrm{~mm}$ line between anchors of no pain on the left (0) and worst possible pain on the right (10) - this was bisected by the patient upon being asked to reflect the patient's average NeP severity over the past 7 days. A successful outcome also required that the patient remain on the prescribed medication for the duration of 6 months of follow-up without medication discontinuation due to intolerable adverse effects or inefficacy as perceived by the patient with respect to pain relief. Failure was recorded as discontinuation of pharmacotherapy provided due to inefficacy, intolerance, or inability to achieve $\geq 30 \%$ pain relief after 3 or 6 months.

Our primary outcome measures were selected to be VAS pain severity and medication discontinuation. For the primary outcomes, the primary independent variable was selected 
to be the catastrophizing subscale of the Coping Strategies Questionnaire (CSQ). ${ }^{17}$ A 50 -item questionnaire, the CSQ reliably assesses eight different coping strategies for managing pain - diversion of attention, reinterpretation of pain sensation, coping self-statements, ignoring of sensations, prayer and hoping, catastrophizing, increased behavioral activities, and pain behavior (Table 1). The CSQ was completed for each study visit and performed prior to clinical assessment. The determination of pain severity (VAS pain scores) and continuance of pharmacotherapy took place on each visit of 0,3 , and 6 months when CSQ questionnaires were also completed.

\section{Secondary outcome measures}

Secondary outcomes consisted of mood and sleep assessments, as well as questionnaires to assess patient satisfaction and impression of change in well-being. Each secondary outcome measure was also completed at each study visit prior to clinical assessment. At the baseline and 6-month visits, additional quality of life (European Quality of Life 5 Domains [EQ-5D]), and Karnofsky Performance Scales (KPS) were assessed for each subject. The EQ-5D was measured as described previously ${ }^{36}$ using two sections - the health state in five dimensions with calculation of a single utility score ${ }^{40}$ and the EQ-5D VAS measuring perception of overall health. The KPS, a clinician-rated scale, was used to rate the patient's physical function over the past 7 days $^{41}$ and was scored using percentiles from $100 \%$ (healthy) to $0 \%$ (death), with scores $<80$ signifying the requirement for assistance with daily activities.

There were additional measures studied at the baseline and 6-month visit time-points. Mood was characterized using the Beck Depression Inventory (BDI) ${ }^{42}$ while sleep efficacy was evaluated with the Medical Outcomes Study Sleep Scale (MOSSS). ${ }^{43}$ The BDI analyzes for severity of depressive symptoms with both mental (hopelessness, irritability) and

Table I Coping strategies questionnaire catastrophizing subscale items

5. It is terrible and I feel it is never going to get any better.

II. It is awful and I feel it overwhelms me.

25. I worry all the time about whether it will end.

33. I feel I can't stand it anymore.

Using a 7-point Likert scale for each item, subjects were asked to selfreport how often they used that strategy when they experienced pain, where $0=$ never, $3=$ sometimes, and $6=$ always.

Note: Adapted from The Coping Strategies Questionnaire catastrophizing subscale Rosenstiel and Keefe. ${ }^{17}$ Adapted with permission from Dr Francis Keefe. physical (fatigue, weight change) symptoms.$^{44}$ The MOSSS is a self-reporting sleep measure used to assess six important dimensions of sleep in adults. ${ }^{45}$ The Patient Global Impression of Change (PGIC) ${ }^{46}$ a simple 7-point scale, was used to assess subjective changes in patient well-being with an intervention - the PGIC was administered at the 6-month follow-up visit. The modified Brief Pain Inventory short form (MBPI $)^{47}$ was used to assess the impact and severity of pain upon daily functions through a description of pain relief, pain quality, the patient's perceptions of the causes of pain intensity, and the degree with which pain interferes with function. ${ }^{48}$

We recorded other demographical variables for each subject, including age, duration of pain, sex, numbers of failed previous pharmacological trials for $\mathrm{NeP}$, and presence of other sources of chronic pain.

\section{Tolerability and adverse events}

An adverse event was defined as any noxious, unintended, or unexpected response suspected to have a causal relationship with the pharmacological intervention used. Adverse events were coded using Medical Dictionary for Regulatory Activities (MedDRA; http://www.meddra.org) terminology. Serious adverse events were defined as any life-threatening reaction to intervention requiring hospitalization, additional urgent physician assessment, or resulted in persistent or significant disability. All spontaneously reported and recorded adverse events related to medications were recorded prospectively at each 3 and 6 month follow-up visit. Discontinuations due to adverse events were recorded in each case - the presence of multiple adverse events leading to discontinuation was noted in addition.

\section{Data analysis}

For both primary and secondary outcome measures, we analyzed the latest available time-point (6-month time-point whenever possible) after at least 3 months. We used multiple logistical regression analyses to determine the association between the dichotomous variables of successful pain relief or medication discontinuation with each of the continuous variables (Catastrophizing Subscore, age and pain duration) and the binary variable (sex) (independent variables).

In addition, we performed linear regression to analyze for changes in the NeP VAS score (dependent variable) with potential covariates of CSQ Catastrophizing Subscore, age, pain duration, and sex (independent variables). We scored intolerability and inefficacy of pharmacotherapy separately. $R^{2}$ values were calculated for each comparison made. Classification of the CSQ catastrophizing subscale score as 
high, medium, or low was performed based upon the average value, with values more than the average + one standard deviation classified as high, and with values less than the average - one standard deviation classified as low; remaining values were defined as medium. Following this, we performed chi-square testing to permit comparisons between levels of high, medium, and low levels of catastrophizing and the primary outcome measures of pain severity and medication discontinuation. We defined $\alpha$ to be 0.05 for significance for each case. When data did not follow a normal distribution based upon Shapiro-Wilk testing, comparisons were made using Mann-Whitney $U$ testing.

Secondary outcome measure data was analyzed using correlational regression techniques. Linear regression analyses were performed using CSQ catastrophizing subscale scores as the independent variable and the baseline VAS pain scores, and the last obtained KPS scores, EQ-5D data, MBPI data, BDI scores, and MOSSS total scores (independent variables), with $R^{2}$ values calculated for each comparison made. Logistical regression was performed to determine the association with CSQ catastrophizing subscale scores and the presence of another chronic pain condition other than the one considered. Finally, we performed a post-hoc comparison of outcomes for the patients prescribed gabapentinoids as compared to those prescribed tricyclic antidepressants for medication success and discontinuation using single factor ANOVA testing. Bonferroni corrections were applied in situations where several independent tests were being performed simultaneously.

For other subscales of the CSQ, we also analyzed for potential associations for subscale baseline values with successful pain relief outcomes and for medication discontinuation using methods identical to those described above.

PGIC scales were analyzed between cohorts at the final follow-up visit time-point using modified Relative to an Identified Distribution (ridit) transformation with the Cochran-Mantel-Haenszel procedure, with adjustment for center; this permits the comparison of grouped, stratified, categorical responses. Data concerning serious adverse effects and presence of comorbidities were tabulated.

\section{Results}

\section{Demographics and interventions}

A total of 66 patients were assessed for eligibility at the time of enrollment (Figure 1). Four patients did not participate in the study after their baseline visit and declined return visit invitations - these patients had idiopathic polyneuropathy in two cases and diabetic polyneuropathy in two cases, were aged $61 \pm 4$ years, had VAS pain severity of $8.1 \pm 1.1$, and CSQ catastrophizing scores of $20.2 \pm 2.8$ at baseline. A total of 62 patients had data analyzed for the 3-month time-point (Table 2), while 58 patients had 6-month time-point data analyzed (four patients were lost to follow-up - three of these patients had diabetic polyneuropathy while one had idiopathic polyneuropathy). Patients lost to follow-up were aged $59 \pm 5$ years, had VAS pain severity of $7.1 \pm 1.0$, and CSQ catastrophizing scores of $18.9 \pm 3.0$ at baseline. Ethnicity was Caucasian in 62/66 (94\%) of patients evaluated. Of the first-line pharmacotherapies provided to enrolled patients (Table 3), amitriptyline was provided most frequently, while nortriptyline and pregabalin were provided least frequently. In the case of pregabalin, the main reason for lack of prescription was inadequate insurance coverage. For the four patients that did not complete the 6-month visit and questionnaires, the 3-month data was used to permit final data analysis using the last value carried forward technique.

\section{Primary outcome measure}

The score for the CSQ subscale for catastrophizing (Table 4) suggested moderate levels of overall catastrophizing. At the individual time-points of 3 and 6 months, there were no remarkable changes seen in CSQ subscale scores when compared to baseline scores, although trends of improvement were noted for the "Ignore pain" sensations, "Increase activity level", and "Control over pain" subscales within the CSQ. The presence of a greater baseline Catastrophizing Subscore within the CSQ was correlated with greater risk of medication discontinuance $\left(\chi^{2}=27.9, P<0.01\right)$ and less likelihood of success ( $>30 \%$ pain relief) with medication intervention $\left(\chi^{2}=27.5, P<0.01\right)$ (Table 5 and Figure 2$)$. There was no association between success with medication intervention and age $\left(\chi^{2}=0.4, P=0.55\right)$ or $\operatorname{sex}\left(\chi^{2}=0.3, P=0.56\right)$, but shorter pain durations were associated with medication success $\left(\chi^{2}=22.6, P<0.01\right)$. Also, there was no association between medication discontinuation and age $\left(\chi^{2}=0.0, P=0.90\right)$ or sex $\left(\chi^{2}=0.7, P=0.42\right)$, but longer durations of pain were associated with greater likelihood of medication discontinuation $\left(\chi^{2}=12.8, P<0.01\right)$. Chi-square testing also revealed significant relationships between a high classification for the CSQ Catastrophizing Subscore and pharmacotherapy discontinuation $(U=319.5 ; P<0.001)$ as well as with failure to achieve $\geq 30 \%$ pain relief $(U=279.0 ; P<0.01)$. There was a strong linear association between the CSQ Catastrophizing Subscore and the change in the VAS pain severity score $\left(R^{2}=0.69, F=129.4, P<0.01\right)$. 


\section{Assessment of the importance of catastrophizing and coping strategies for successful}

treatment of peripheral neuropathic pain

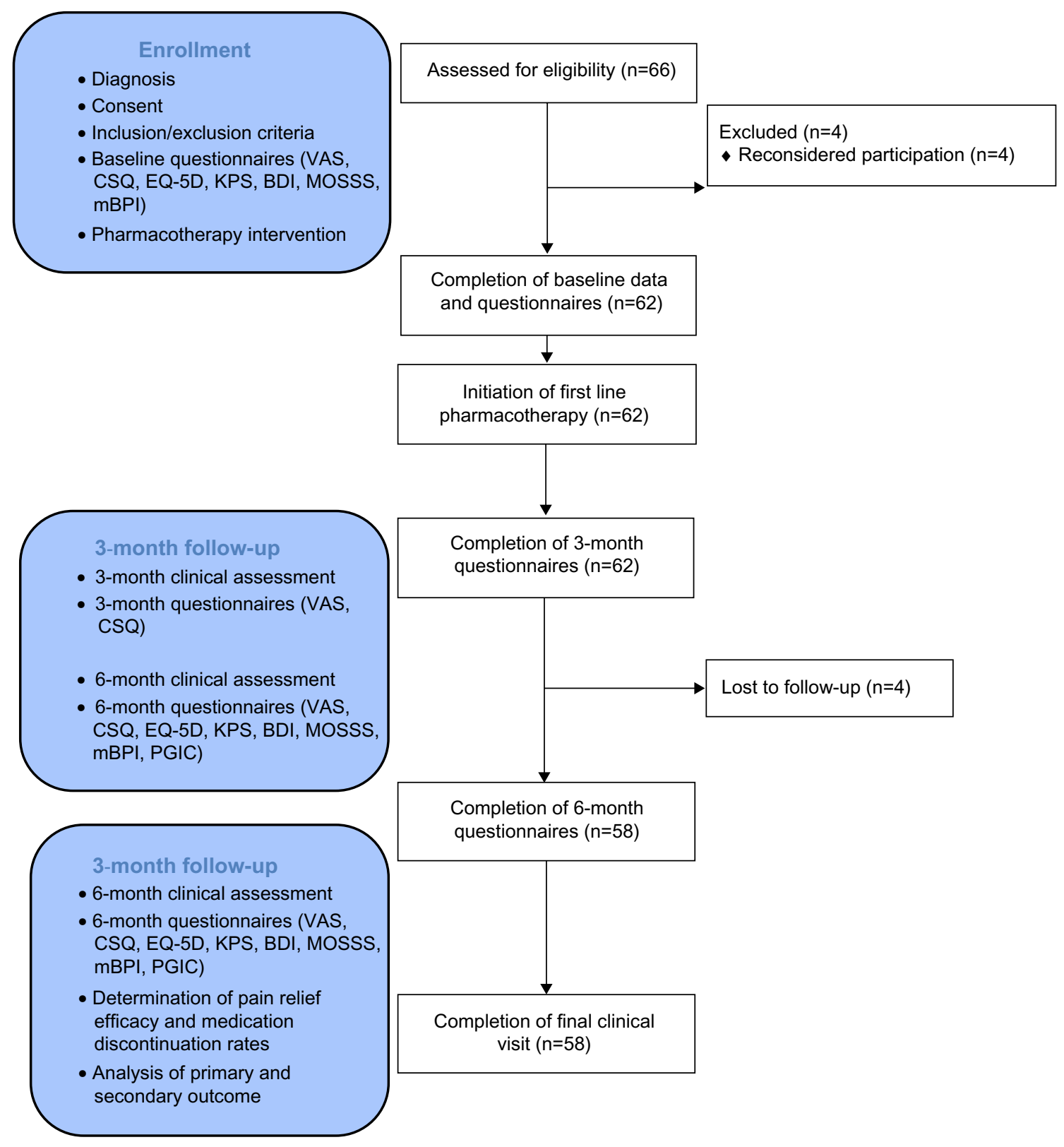

Figure I The CONSORT flow diagram for patients evaluated in this study.

Abbreviations: BDI, Beck Depression Inventory; CSQ, Coping Strategies Questionnaire; EQ-5D, European Quality of Life 5 Domains; KPS, Karnofsky Performance Scales; mBPI, modified Brief Pain Inventory short form; MOSSS, Medical Outcomes Study Sleep Scale; PGIC, Patient Global Impression of Change; VAS, visual analog scale.

Pain severity levels, measured using the VAS, improved at the 3-month follow-up period by $1.6 \pm 1.2$ and at the 6-month follow-up period by $2.5 \pm 1.4$ overall. Improvement in pain severity was related to the primary outcome factor, the CSQ catastrophizing subscale score (Figure 2).

\section{Secondary outcome measures}

Reported quality of life (EQ-5D) index scores $\left(R^{2}=0.54\right.$, $F=67.7, P<0.01)$ and disability (KPS) scores $\left(R^{2}=0.59\right.$,
$F=84.9, P<0.01)$ also correlated with the CSQ catastrophizing subscale score based upon baseline values but did not demonstrate change over time. There was also a significant relationship between the numbers of previously failed pharmacological trials and the CSQ catastrophizing subscale score $\left(R^{2}=0.36, F=42.2, P<0.01\right)$. Also, there were no significant changes in the mood (BDI total score), sleep efficacy (MOSSS Sleep Problems Index) score, or the MBPI subscale (general activity, mood, walking ability, relationships, sleep, 
Table 2 Patient characteristics for all patients enrolled to determine catastrophizing or coping skills

\begin{tabular}{ll}
\hline & $\begin{array}{l}\text { Patient characteristics } \\
(\mathbf{n = 6 2 )}\end{array}$ \\
\hline Sex: females (\%) & $36(58 \%)$ \\
Age (years [range]) & $51.9 \pm 7.2[34-86]$ \\
Duration of NeP (years) & $6.7 \pm 3.4[1.5-7.8]$ \\
Number of pain medications previously & $1.6 \pm 1.2[0-4]$ \\
failed & \\
Diagnosis & 27 \\
$\quad$ Diabetic polyneuropathy & 22 \\
Idiopathic polyneuropathy & 5 \\
Vitamin deficiency polyneuropathy & 4 \\
Polyneuropathy associated with & \\
monoclonal gammopathy & 2 \\
Inflammatory polyneuropathy & 1 \\
Vasculitic polyneuropathy & 1 \\
Hereditary polyneuropathy &
\end{tabular}

Notes: Values expressed are means \pm standard deviations, with numbers in square brackets representing range of values and numbers in rounded brackets representing percentages.

normal work, or enjoyment) scores for all patients. Finally, we did not find any correlations between the CSQ catastrophizing subscale scores and each of the BDI total score $\left(R^{2}=0.11\right.$, $F=4.8, P=0.27)$, MOSSS Sleep Problems Index $\left(R^{2}=0.14\right.$, $F=5.4, P=0.22)$, or MBPI subscale scores $\left(R^{2}=0.06-0.19\right.$, $F=2.6-7.6, P=0.08-0.51)$, and presence of other sources of chronic pain $\left(\chi^{2}=3.8, P=0.24\right)$. We classified the CSQ catastrophizing subscale score as high, medium, or low as described for the assessment of the PGIC. Those patients with high CSQ catastrophizing subscale scores had significantly more negative outcomes on the PGIC than those with low CSQ catastrophizing subscale scores (Figure 3).

The baseline values of the other subscales of the CSQ were analyzed in addition to the catastrophizing subscale score. The following subscales did not demonstrate association with either medication success or medication discontinuation: reinterpret pain sensations $\left(\chi^{2}=1.5-1.8, P=0.29-0.33\right)$, coping self-statements $\left(\chi^{2}=0.8-1.3, P=0.42-0.53\right)$, ignore pain sensations $\left(\chi^{2}=0.9-1.6, P=0.31-0.46\right)$, control over pain $\left(\chi^{2}=2.1-2.5, P=0.13-0.16\right)$, and ability to decrease pain $\left(\chi^{2}=1.2-1.4, P=0.44-0.51\right)$. The following subscales had negative associations with medication success: diverting attention $\left(\chi^{2}=4.7, P=0.03\right)$, praying or hoping $\left(\chi^{2}=6.9\right.$, $P=0.01)$, and increase activity level $\left(\chi^{2}=6.1, P=0.02\right)$. On the other hand, some subscales were positively associated with medication continuation: praying or hoping $\left(\chi^{2}=8.2, P<0.01\right)$ and increase activity level $\left(\chi^{2}=6.5, P=0.01\right)$, while diverting attention was not significantly associated with medication continuation $\left(\chi^{2}=3.4, P=0.07\right)$.

Finally, there was no difference in outcomes for patients prescribed gabapentinoids as compared to those prescribed tricyclic antidepressants for either of medication success (ANOVA, $F=0.97, P=0.33$ ) or medication discontinuations (ANOVA, $F=0.04, P=0.85$ ).

\section{Discussion}

In this study, the negative coping skill of catastrophizing was shown to impact upon pharmacotherapy-mediated outcomes in patients with chronic $\mathrm{NeP}$ and was associated with a greater chance of pharmacotherapy inefficacy and discontinuation. Higher levels of catastrophizing were also associated with greater levels of self-reported disability, lower quality of life scores, longer duration of painful conditions, and poor global impression of change scores. These results suggest that pre-intervention screening may be useful to identify patients with higher levels of catastrophizing in order to prevent unnecessary exposure to pharmacotherapies postulated to be of little benefit.

Our results are similar to other studies examining the role of catastrophizing upon physical therapy or topical analgesic interventions. ${ }^{30-32}$ Most previous work has demonstrated a strong positive relationship between high catastrophizing scores and more severe pain levels. ${ }^{22}$ Also, although we examined patients with peripheral neuropathy and associated $\mathrm{NeP}$, other conditions causing $\mathrm{NeP}$ also have important relationships with catastrophizing, including post-herpetic neuralgia, ${ }^{33}$ spinal cord injury, ${ }^{49}$ and phantom limb pain. ${ }^{50}$ Our results extend upon these previous studies showing the clinical importance of catastrophizing upon pharmacological outcomes using the most commonly prescribed oral medications for NeP relief. If not recognized, high catastrophizing may lead to dosing escalations being performed in patients leading to greater analgesic intake, ${ }^{51}$ which may increase

Table 3 Medication interventions provided to patients

\begin{tabular}{llcc}
\hline & $\begin{array}{l}\text { Number of patients } \\
\text { receiving specific medication }\end{array}$ & $\begin{array}{l}\text { Initial medication } \\
\text { daily dose }\end{array}$ & $\begin{array}{l}\text { Final medication } \\
\text { daily dose }\end{array}$ \\
\hline Amitryptyline & $25(40 \%)$ & $15.0 \pm 5.2 \mathrm{mg}$ & $37.3 \pm 12.7 \mathrm{mg}$ \\
Nortryptyline & $10(16 \%)$ & $25.5 \pm 11.3 \mathrm{mg}$ & $55.7 \pm 13.3 \mathrm{mg}$ \\
Gabapentin & $17(27 \%)$ & $667.0 \pm 112.3 \mathrm{mg}$ & $5 / 10(50 \%)$ \\
Pregabalin & $10(16 \%)$ & $120.5 \pm 62.3 \mathrm{mg}$ & $7 / 562.7 \pm 147.8 \mathrm{mg}$ \\
\hline
\end{tabular}

Note: Values expressed are means \pm standard deviations, with numbers in rounded brackets representing percentages. 
Table 4 Coping strategies questionnaire subscale results

\begin{tabular}{|c|c|c|c|}
\hline Measure & $\begin{array}{l}\text { Baseline scores } \\
(n=66)\end{array}$ & $\begin{array}{l}\text { 3-month scores } \\
(n=62)\end{array}$ & $\begin{array}{l}\text { 6-month scores } \\
(n=58)\end{array}$ \\
\hline Diverting attention (/36) & $11.9 \pm 6.2[4,36](13)$ & II.5 $5 \pm 5.8[4,36](12)$ & 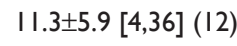 \\
\hline Reinterpret pain sensations (/36) & $6.4 \pm 4.8[2,22](8)$ & $6.7 \pm 4.5[2,21](8)$ & $6.6 \pm 4.6[2,22](8)$ \\
\hline Coping self-statements (/36) & $20.3 \pm 7.6[3,36](19)$ & $20.8 \pm 7.2[4,36](20)$ & $20.5 \pm 8.0[4,36](20)$ \\
\hline Ignore pain sensations (/36) & $16.8 \pm 7.3[3,30](18)$ & $16.5 \pm 6.5[3,28](17)$ & $15.8 \pm 5.7[3,29](16)$ \\
\hline Praying or hoping (/36) & $10.9 \pm 6.0[0,35](9)$ & $10.7 \pm 5.7[0,36](10)$ & $10.8 \pm 5.9[0,36](10)$ \\
\hline Catastrophizing (/36) & $15.5 \pm 7.0[2,36](15)$ & $15.3 \pm 6.7[3,36](15)$ & $15.4 \pm 6.5[2,36](15)$ \\
\hline Increase activity level (/36) & $17.3 \pm 7.4[4,36](17)$ & $16.9 \pm 6.5[5,36](18)$ & $15.9 \pm 5.4[6,36](17)$ \\
\hline Control over pain (/36) & $3.9 \pm 2.2[0,20](5)$ & $3.6 \pm 2.0[0,22](5)$ & $3.5 \pm 2.0[0,22](5)$ \\
\hline Ability to decrease pain (/36) & $2.9 \pm 1.8[0,18](4)$ & $2.7 \pm 1.7[0,22](4)$ & $3.1 \pm 1.9[0,22](4)$ \\
\hline
\end{tabular}

Notes: Values expressed are means \pm standard deviations, with numbers in square brackets representing the range of values and numbers in rounded brackets representing the median. Scores for each subscale are out of a total of 36 possible points.

adverse effects and risks of toxicity. Furthermore, the entry of patients with high catastrophizing levels likely impacts upon the results of clinical trials, adding to uncertainty with observed outcomes. Greater clinical recognition of catastrophizing could influence levels of pharmacotherapy exposure, and permit psychological and rehabilitative interventions to be considered. ${ }^{27,52}$

It has become clear that catastrophizing is significantly associated with both pain and pain-related disability independent of depression. ${ }^{53-58}$ It is possible that catastrophizing disrupts endogenous pain pathways,${ }^{59}$ perhaps through loss of inhibitory controls. The development and subsequent maintenance of catastrophizing may relate to social goals and psychosocial factors developed through life. ${ }^{22}$ Catastrophizing may increase pain-related fear, thus increasing attention being paid to the stimuli. In catastrophizers, a vicious circle may result in which catastrophizing thoughts lead to avoidance of activity, further propagating deconditioning and leading to failure to return to work and regular life.

Our greater recognition of the impact of negative coping skills has led to enhanced appreciation for psychosocial treatments focusing on pain-coping strategies. Such non-pharmacological treatments may include cognitive behavioral therapy strategies which can assist with achieving three main components for successfully coping with pain: 1) an educational approach documenting the influence of patients' thoughts, feelings, and behaviors upon pain and pain coping; 2) training in cognitive and behavioral-coping strategies, such as with relaxation, setting appropriate goals, and cognitive restructuring; and 3) education for home-coping skills. ${ }^{60}$

There are a number of limitations associated with our results. First, this was a small patient cohort treated at one interdisciplinary clinic - outcomes may not be generalizable to primary care clinics or other tertiary clinics. Although we chose to analyze 3- and 6-month time-points, this may not be a sufficient duration of time to determine possible changes in coping skills. We did not perform interventions for coping skills in this study designed to assess pharmacological responses. We did not anticipate large scales changes in catastrophizing scores, but the absence of any significant change in the CSQ catastrophizing subscale score after improvement in pain severity was unexpected as previous studies have

Table 5 There were significant correlations between the catastrophizing subscale score of the coping strategies questionnaire and with medication discontinuance and medication success

\begin{tabular}{|c|c|c|c|c|c|c|c|}
\hline \multirow[t]{2}{*}{ Factor } & \multirow[t]{2}{*}{ Measure } & Factor & \multirow[t]{2}{*}{ I } & \multirow[t]{2}{*}{2} & \multirow[t]{2}{*}{3} & \multirow[t]{2}{*}{4} & \multirow[t]{2}{*}{5} \\
\hline & & Mean \pm SD & & & & & \\
\hline I & VAS pain intensity & $61.1 \pm 8.8$ & & & & & \\
\hline 2 & EQ-5D disability index score & $0.62 \pm 0.11$ & $-0.22^{\mathrm{a}}$ & & & & \\
\hline 3 & $\begin{array}{l}\text { Catastrophizing subscale score of the } \\
\text { Coping Strategies Questionnaire (/36) }\end{array}$ & $15.5 \pm 7.0$ & -0.17 & $0.30^{\mathrm{a}}$ & & & \\
\hline 4 & Medication continuance & $32 / 62(52 \%)$ & 0.08 & 0.15 & $-0.36^{\mathrm{a}}$ & & \\
\hline 5 & Medication success & $21 / 62(34 \%)$ & 0.13 & 0.11 & $-0.34^{a}$ & $0.54^{\mathrm{a}}$ & \\
\hline 6 & Karnofsky Performance Scales & $77.6 \pm 9.7$ & -0.23 & 0.21 & $-0.34^{a}$ & 0.12 & 0.13 \\
\hline
\end{tabular}

Notes: Secondary outcome measures of interest ([EQ-5D] and Karnofsky Performance Scale) also possessed significant relationships (aP $<0.05$, Pearson product-moment correlations). Values expressed are means \pm standard deviations (SD) and represent data obtained at the 6-month follow-up visit in the third column. Remaining columns present the $r$ value for correlation.

Abbreviations: EQ-5D, European Quality of Life 5 Domains; VAS, visual analog scale. 
A

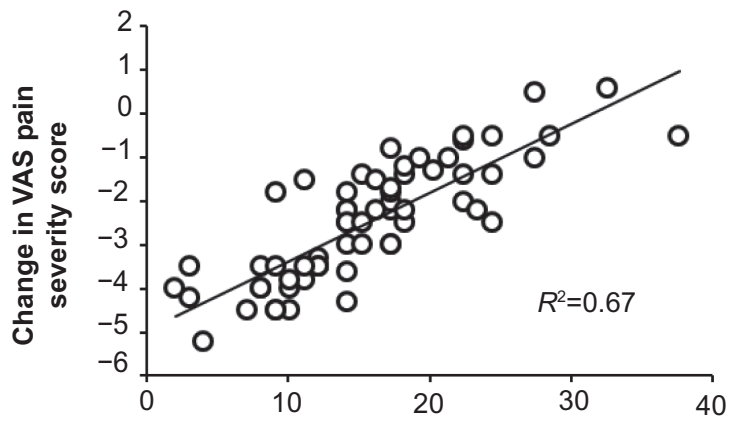

C

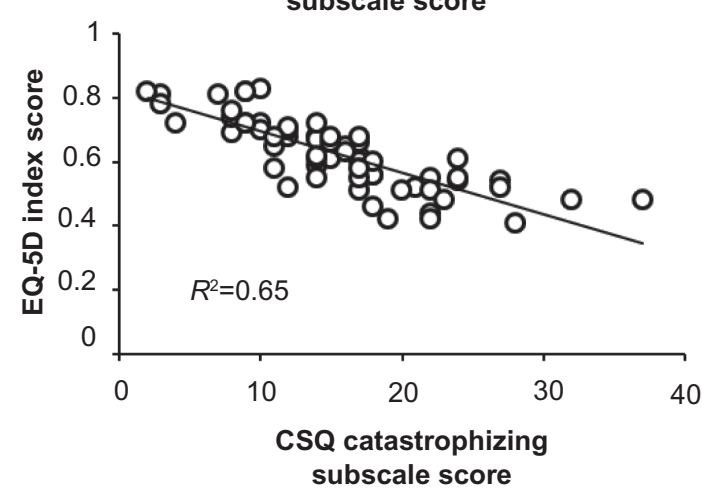

B

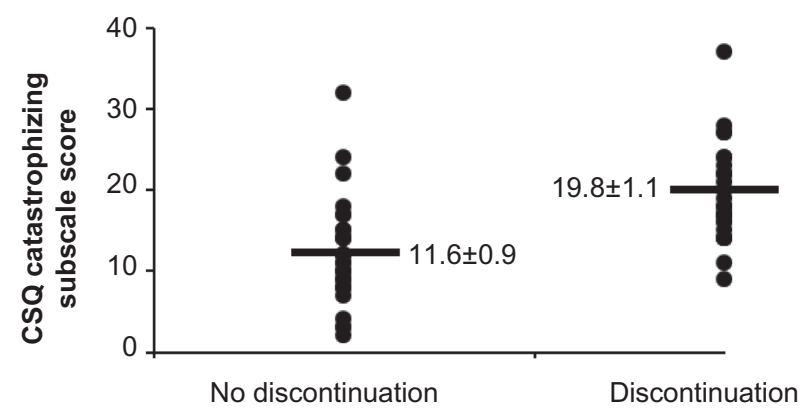

D

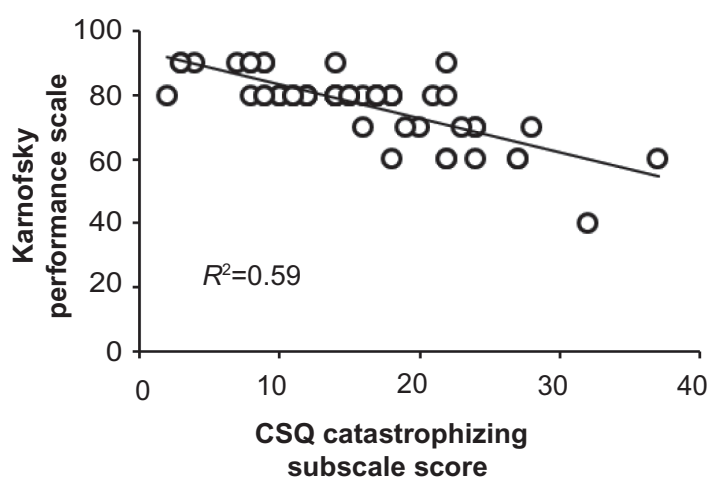

Figure 2 Correlational analyses for the Coping Strategies Questionnaire (CSQ) catastrophizing subscale score are demonstrated.

Notes: The change in the primary outcome measure pain severity score (evaluated using the visual analog scale [VAS]) had a positive relationship with CSQ catastrophizing subscale scores, with a negative change in the VAS reflecting improvement in pain severity (A) (Spearman's rho, $P<0.05$ ). The presence of medication discontinuation by final endpoint was associated with significantly greater CSQ catastrophizing subscale scores (see Table 5) (B). There were negative significant relationships for CSQ catastrophizing subscale scores with both of the EuroQol Quality of Life -5 Domains (EQ-5D) Index Score (C) (Spearman rho, $P<0.05)$ and for the Karnofsky Performance Scale (D) (Spearman rho, $P<0.05$ ). $R^{2}$ values for each correlational analysis are provided on each subfigure. In subfigure $2 \mathrm{~B}$, horizontal lines indicate average values for the CSQ catastrophizing subscale scores for each subgroup, with numbers provided demonstrating averages \pm standard errors.

demonstrated reductions in patient catastrophizing scale scores after lengthy interventions. ${ }^{32}$ It should be noted that the CSQ catastrophizing subscale scores in our patient population were in a low-moderate range when compared to other published works. ${ }^{32,53,61,62}$ We selected the CSQ catastrophizing subscale in order to determine the primary outcome measure, but a number of pain-related catastrophizing scales have been developed (but not used in our study) including the Cognitive Error Questionnaire, ${ }^{63}$ the Pain Anxiety Symptoms Scale, ${ }^{64}$ the Cognitive Errors Questionnaire catastrophizing subscale, ${ }^{63}$ and the Pain Catastrophizing Scale. ${ }^{21}$ We did not demonstrate any influence of age ${ }^{65}$ or $\operatorname{sex}^{66}$ upon catastrophizing in the present study, although these factors were important in other studies. There was insufficient heterogeneity in
A

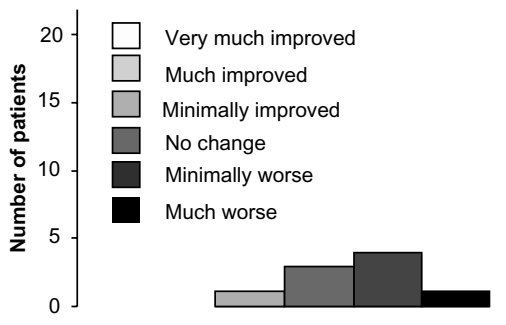

B

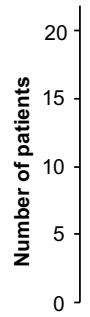

C

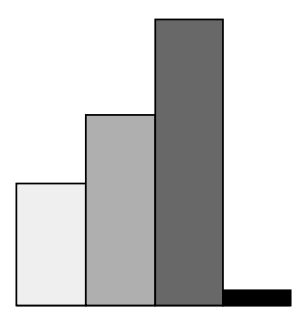

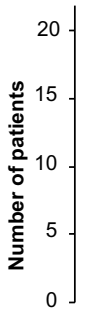

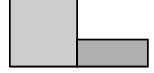

Figure 3 Patient global impression of change (PGIC) was reported at the 6-month time-point, demonstrating significantly less perceived benefit for scores obtained from "high" catastrophizers (A) as compared to "low" catastrophizers (C) (modified ridit transformation with the Cochran-Mantel-Haenszel procedure, adjusting for center, *P<0.05), with "medium" catastrophizers shown in (B). There were no significant differences between "high" and "medium" catastrophizers, or between "low" and "medium" catastrophizers. 
ethnicity to evaluate its effects upon catastrophizing. While all patients were encouraged to use conservative measures to assist with $\mathrm{NeP}$ relief including the pharmacotherapeutic intervention provided, there was no method by which to control for non-pharmacological interventions, or other invasive or non-invasive interventions received at other health care centers. Also, we could not control the use of additional over-the-counter medications that were unreported. There were multiple first-line pharmacological options offered in the study which may have differentially affected coping skills. Finally, patients referred to this tertiary care clinic may not represent the general population of patients with NeP, as not all chronic pain patients will accept referral to a multidisciplinary clinic. ${ }^{67}$

\section{Conclusion}

Our results suggest that the presence of heightened catastrophizing modulates pharmacotherapy tolerance or efficacy for patients with NeP due to polyneuropathy. The scales used to capture this data are easily performed in outpatient primary or tertiary care clinics. In patients presenting with higher levels of catastrophizing, therapies other than that of standard pharmacotherapies may be more advantageous. The additional use of psychosocial assessments and evaluations of coping skills may be of greater utility for this particular patient subpopulation.

\section{Acknowledgment}

This study was supported by an unrestricted grant through the Department of Clinical Neurosciences and the Division of Neurology.

\section{Author contributions}

CT developed the concept, devised the protocol and ethics approval, assisted in data analysis, and wrote the manuscript. $\mathrm{SB}$ and $\mathrm{MH}$ assisted with questionnaire completion and data analysis, and both have read the manuscript and edited the final submitted manuscript.

\section{Disclosure}

Dr Toth received salary support from the Alberta Heritage Foundation for Medical Research. Dr Toth has received research funding from Pfizer Canada, Lilly International, Valeant Canada, CSL Behring, and Baxter Ltd. The authors have no other conflicts of interest in this work.

\section{References}

1. International Association for the Study of Pain [webpage on the Internet]. IASP Taxonomy. Washington, DC: International Association for the Study of Pain; 2012 [updated May 22, 2012]. Available from: http:// www.iasp-pain.org/Content/NavigationMenu/GeneralResourceLinks/ PainDefinitions/default.htm\#Neuropathicpain. Accessed April 12, 2012.
2. Mauskopf J, Austin R, Dix L, Berzon R. The Nottingham Health Profile as a measure of quality of life in zoster patients: convergent and discriminant validity. Qual Life Res. 1994;3(6):431-435.

3. Meyer-Rosberg K, Kvarnstrom A, Kinnman E, Gordh T, Nordfors LO, Kristofferson A. Peripheral neuropathic pain - a multidimensional burden for patients. Eur J Pain. 2001;5(4):379-389.

4. Gordon A, Choiniere M, Collet JP. The humanistic burden of neuropathic pain in Canada. J Outcomes Res. 2006;10:23-35.

5. McDermott AM, Toelle TR, Rowbotham DJ, Schaefer CP, Dukes EM. The burden of neuropathic pain: results from a cross-sectional survey. Eur J Pain. 2006;10(2):127-135.

6. Gourlay GK, Cherry DA, Cousins MJ. A comparative study of the efficacy and pharmacokinetics of oral methadone and morphine in the treatment of severe pain in patients with cancer. Pain. 1986;25(3): 297-312.

7. Persson AK, Gebauer M, Jordan S, et al. Correlational analysis for identifying genes whose regulation contributes to chronic neuropathic pain. Mol Pain. 2009;5:7.

8. Gore M, Sadosky A, Stacey BR, Tai KS, Leslie D. The burden of chronic low back pain: clinical comorbidities, treatment patterns, and health care costs in usual care settings. Spine (Phila Pa 1976). 2012;37(11):E668-E677.

9. Free MM. Cross-cultural conceptions of pain and pain control. Proc (Bayl Univ Med Cent). 2002;15(2):143-145.

10. Baetz M, Bowen R. Chronic pain and fatigue: Associations with religion and spirituality. Pain Res Manag. 2008;13(5):383-388.

11. Melzack R, Wall PD. Pain mechanisms: a new theory. Science. 1965;150(3699):971-979.

12. Jensen MP, Turner JA, Romano JM, Karoly P. Coping with chronic pain: a critical review of the literature. Pain. 1991;47(3):249-283.

13. Binzer M, Almay B, Eisemann M. Chronic pain disorder associated with psychogenic versus somatic factors: a comparative study. Nordic J Psychiatry. 2003;57(1):61-66.

14. Lazarus R, Folkman S. Stress, Appraisal and Coping. New York: Springer; 1984.

15. Skinner EA, Edge K, Altman J, Sherwood H. Searching for the structure of coping: a review and critique of category systems for classifying ways of coping. Psychol Bull. 2003;129(2):216-269.

16. Van Damme S, Crombez G, Eccleston C. Coping with pain: a motivational perspective. Pain. 2008;139(1):1-4.

17. Rosenstiel AK, Keefe FJ. The use of coping strategies in chronic low back pain patients: relationship to patient characteristics and current adjustment. Pain. 1983;17(1):33-44.

18. Turner JA, Clancy S. Strategies for coping with chronic low back pain: relationship to pain and disability. Pain. 1986;24(3):355-364.

19. Fernandez E, Turk DC. The utility of cognitive coping strategies for altering pain perception: a meta-analysis. Pain. 1989;38(2): 123-135.

20. Brown GK, Nicassio PM. Development of a questionnaire for the assessment of active and passive coping strategies in chronic pain patients. Pain. 1987;31(1):53-64.

21. Sullivan MJL, Bishop S, Pivik J. The Pain Catastrophizing Scale: development and validation. Psychol Assess. 1995;7:524-532.

22. Sullivan MJ, Thorn B, Haythornthwaite JA, et al. Theoretical perspectives on the relation between catastrophizing and pain. Clin J Pain. 2001;17(1):52-64.

23. Griswold GA, Evans S, Spielman L, Fishman B. Coping strategies of HIV patients with peripheral neuropathy. AIDS care. 2005;17(6): 711-720.

24. Parr JJ, Borsa PA, Fillingim RB, et al. Pain-related fear and catastrophizing predict pain intensity and disability independently using an induced muscle injury model. J Pain. 2012;13(4):370-378.

25. Edwards RR, Cahalan C, Mensing G, Smith M, Haythornthwaite JA. Pain, catastrophizing, and depression in the rheumatic diseases. Nat Rev Rheumatol. 2011;7(4):216-224.

26. Sullivan M, Bishop S, Pivik J. The pain catastrophizing scale: development and validation. Psychol Assess. 1995;7:524-532. 
27. Sullivan MJ, Adams H, Rhodenizer T, Stanish WD. A psychosocial risk factor - targeted intervention for the prevention of chronic pain and disability following whiplash injury. Phys Ther. 2006;86(1):8-18.

28. Arnow BA, Blasey CM, Constantino MJ, et al. Catastrophizing, depression and pain-related disability. Gen Hosp Psychiatry. 2011;33(2): $150-156$.

29. Lucey BP, Clifford DB, Creighton J, Edwards RR, McArthur JC, Haythornthwaite J. Relationship of depression and catastrophizing to pain, disability, and medication adherence in patients with HIVassociated sensory neuropathy. AIDS care. 2011;23(8):921-928.

30. Slepian P, Bernier E, Scott W, Niederstrasser NG, Wideman T, Sullivan M. Changes in pain catastrophizing following physical therapy for musculoskeletal injury: the influence of depressive and posttraumatic stress symptoms. J Occup Rehabil. 2013;23(1):63-73.

31. Mankovsky T, Lynch M, Clark A, Sawynok J, Sullivan MJ. Pain catastrophizing predicts poor response to topical analgesics in patients with neuropathic pain. Pain Res Manag. 2012;17(1):10-14.

32. Scott W, Wideman TH, Sullivan MJ. Clinically meaningful scores on pain catastrophizing before and after multidisciplinary rehabilitation: a prospective study of individuals with subacute pain after whiplash injury. Clin J Pain. Epub April 1, 2013.

33. Haythornthwaite JA, Clark MR, Pappagallo M, Raja SN. Pain coping strategies play a role in the persistence of pain in post-herpetic neuralgia. Pain. 2003;106(3):453-460.

34. Nicholas MK, Asghari A, Corbett M, et al. Is adherence to pain selfmanagement strategies associated with improved pain, depression and disability in those with disabling chronic pain? Eur J Pain. 2012;16(1): 93-104.

35. Moulin DE, Clark AJ, Gilron I, et al. Pharmacological management of chronic neuropathic pain - consensus statement and guidelines from the Canadian Pain Society. Pain Res Manag. 2007;12(1):13-21.

36. Garven A, Brady S, Wood S, et al. The impact of enrollment in a specialized interdisciplinary neuropathic pain clinic. Pain Res Manag. 2011;16(3):159-168.

37. Bouhassira D, Attal N, Alchaar H, et al. Comparison of pain syndromes associated with nervous or somatic lesions and development of a new neuropathic pain diagnostic questionnaire (DN4). Pain. 2005;114(1-2): 29-36.

38. Poliakov I, Toth C. The impact of pain in patients with polyneuropathy. Eur J Pain. 2011;15(10):1015-1022.

39. Moulin DE, Clark AJ, Gilron I, et al. Pharmacological management of chronic neuropathic pain - consensus statement and guidelines from the Canadian Pain Society. Pain Res Manag. 2007;12(1):13-21.

40. Kind P, Dolan P, Gudex C, Williams A. Variations in population health status: results from a United Kingdom national questionnaire survey. BMJ. 1998;316(7133):736-741.

41. Mor V, Laliberte L, Morris JN, Wiemann M. The Karnofsky Performance Status Scale. An examination of its reliability and validity in a research setting. Cancer. 1984;53(9):2002-2007.

42. Joe S, Woolley ME, Brown GK, Ghahramanlou-Holloway M, Beck AT. Psychometric properties of the Beck Depression Inventory-II in low-income, African American suicide attempters. J Pers Assess. 2008;90(5):521-523.

43. Stewart AL, Ware JE. Measuring functioning and well-being: The Medical Outcomes Study approach. In: Hays RD, Stewart AL, editors. Sleep Measures. Durham, NC: Duke University Press; 1992:235-259.

44. Beck AT, Ward CH, Mendelson M, Mock J, Erbaugh J. An inventory for measuring depression. Arch Gen Psychiatry. 1961;4:561-571.

45. Hays RD, Stewart AL. Sleep measures. In: Stewart AL, Ware JE, editors. Measuring Functioning and Well-being: The Medical Outcomes Study Approach. Durham, NC: Duke University Press; 1992:235-259.

46. Hurst H, Bolton J. Assessing the clinical significance of change scores recorded on subjective outcome measures. J Manipulative Physiol Ther. 2004;27(1):26-35
47. Zelman DC, Gore M, Dukes E, Tai KS, Brandenburg N. Validation of a modified version of the brief pain inventory for painful diabetic peripheral neuropathy. J Pain Symptom Manage. 2005;29(4):401-410.

48. Zelman DC, Gore M, Dukes E, Tai KS, Brandenburg N. Validation of a modified version of the Brief Pain Inventory for painful diabetic peripheral neuropathy. J Vasc Nurs. 2005;23(3):97-104.

49. Turner JA, Jensen MP, Warms CA, Cardenas DD. Catastrophizing is associated with pain intensity, psychological distress, and pain-related disability among individuals with chronic pain after spinal cord injury. Pain. 2002;98(1-2):127-134.

50. Jensen MP, Ehde DM, Hoffman AJ, Patterson DR, Czerniecki JM, Robinson LR. Cognitions, coping and social environment predict adjustment to phantom limb pain. Pain. 2002;95(1-2):133-142.

51. Jacobsen PB, Butler RW. Relation of cognitive coping and catastrophizing to acute pain and analgesic use following breast cancer surgery. J Behav Med. 1996;19(1):17-29.

52. Smeets RJ, Vlaeyen JW, Kester AD, Knottnerus JA. Reduction of pain catastrophizing mediates the outcome of both physical and cognitivebehavioral treatment in chronic low back pain. J Pain. 2006;7(4): 261-271.

53. Keefe FJ, Brown GK, Wallston KA, Caldwell DS. Coping with rheumatoid arthritis pain: catastrophizing as a maladaptive strategy. Pain. 1989;37(1):51-56.

54. Keefe FJ, Caldwell DS, Williams DA, et al. Pain coping skills training in the management of osteoarthritic knee pain II: follow-up results. Behav Ther. 1990;21:435-447.

55. Geisser ME, Robinson ME, Keefe FJ, Weiner ML. Catastrophizing, depression and the sensory, affective and evaluative aspects of chronic pain. Pain. 1994;59(1):79-83.

56. Geisser ME, Casey KL, Brucksch CB, Ribbens CM, Appleton BB, Crofford LJ. Perception of noxious and innocuous heat stimulation among healthy women and women with fibromyalgia: association with mood, somatic focus, and catastrophizing. Pain. 2003;102(3):243-250.

57. Geisser ME, Roth RS. Knowledge of and agreement with pain diagnosis: relation to pain beliefs, pain severity, disability, and psychological distress. J Occup Rehabil. 1998;8:73-88.

58. Sullivan MJ, Stanish W, Waite H, Sullivan M, Tripp DA. Catastrophizing, pain, and disability in patients with soft-tissue injuries. Pain. 1998;77(3):253-260.

59. Goodin BR, McGuire L, Allshouse M, et al. Associations between catastrophizing and endogenous pain-inhibitory processes: sex differences. J Pain. 2009;10(2):180-190.

60. Keefe FJ, Somers TJ, Martire LM. Psychologic interventions and lifestyle modifications for arthritis pain management. Rheum Dis Clin North Am. 2008;34(2):351-368.

61. Keefe FJ, Williams DA. A comparison of coping strategies in chronic pain patients in different age groups. J Gerontol. 1990;45(4):P161-P165.

62. Michael ES, Burns JW. Catastrophizing and pain sensitivity among chronic pain patients: moderating effects of sensory and affect focus. Ann Behav Med. 2004;27(3):185-194.

63. Lefebvre MF. Cognitive distortion and cognitive errors in depressed psychiatric and low back pain patients. J Consult Clin Psychol. 1981; 49(4):517-525.

64. McCracken LM, Zayfert C, Gross RT. The Pain Anxiety Symptoms Scale: development and validation of a scale to measure fear of pain. Pain. 1992;50(1):67-73.

65. Ruscheweyh R, Nees F, Marziniak M, Evers S, Flor H, Knecht S. Pain catastrophizing and pain-related emotions: influence of age and type of pain. Clin J Pain. 2011;27(7):578-586

66. Jensen I, Nygren A, Gamberale F, Goldie I, Westerholm P. Coping with long-term musculoskeletal pain and its consequences: is gender a factor? Pain. 1994;57(2):167-172.

67. Turk DC, Okifuji A. Efficacy of multidisciplinary pain centres: an antidote to anecdotes. Bailliere's Clin Anaesthesiol. 1998;12(1):103-119. 
Journal of Pain Research

\section{Dovepress}

\section{Publish your work in this journal}

The Journal of Pain Research is an international, peer-reviewed, open access, online journal that welcomes laboratory and clinical findings in the fields of pain research and the prevention and management of pain. Original research, reviews, symposium reports, hypothesis formation and commentaries are all considered for publication.

The manuscript management system is completely online and includes a very quick and fair peer-review system, which is all easy to use. Visit http://www.dovepress.com/testimonials.php to read real quotes from published authors.

Submit your manuscript here: http://www.dovepress.com/journal-of-pain-research-journal 